\title{
VISIBILITY MONITORING USING MOBILE APPLICATION
}

\author{
Suchit Purohit ${ }^{1, *}$, Prakash Chauhan ${ }^{2}$ \\ ${ }^{1}$ Department of Computer Science, Gujarat University, Ahmedabad, India- suchit.s.purohit@ieee.org \\ ${ }^{2}$ Indian Institute of Remote Sensing, ISRO, Dehradun, India- prakash@iirs.gov.in
}

Commission V: WG V/3

KEY WORDS: Visibility, atmospheric turbidity, Perez model, Android

\begin{abstract}
:
Visibility is clarity with which the distant objects are perceived in the atmosphere with the naked eye. Visibility monitoring is an important concern in health, environment and transport safety context. Quantitative measures of visibility are increasingly becoming important in various areas as they are representative of the particles present in the environment that causes degradation of the visibility. Existing techniques of visibility estimation employ human observers, optical instruments, chemical sensors or combination of some of them. These techniques suffer from poor spatial and temporal resolution, high cost of installation and maintenance, need of specialized personnel, continuous power supply requirement and difficulty in portability. We propose a smart phone-based visibility monitoring system which estimates air visibility/quality in terms of a quantitative measure: Turbidity. In principle, the application calculates turbidity as difference of intensity of captured sky image and analytical value of sky luminance obtained by implementing Perez model. The estimated turbidity tagged with date, time, location, solar position and luminance is sent to the backend server generating consolidated database for mapping of turbidity and generating various analytical reports. The application can easily be deployed to be used by large number of people facilitating citizen science. The results from application were validated against the observations from SAFAR INDIA application at different stations in Ahmedabad, dates and under variable weather conditions.
\end{abstract}

\section{I.INTRODUCTION}

Air quality varies from place to place, as per seasons and according to geography of the place. Both natural phenomena and human activities play a decisive role in defining the quality of air. The main cause of air quality degradation is the atmospheric pollutants in the form of haze aerosols. These are the tiny particles suspended in air changing the hue of the air. Due to the scattering or absorption of sunlight by these pollutants, sky appears to be of different hues and colours. The source of this particulate matter can be natural like dust, fog, smog, haze, volcanic eruption etc., and/or manmade like combustion of fuel, pollution, urbanization, population, etc. Besides hazardous ill effects on health, air quality degradation has a prominent effect on visibility through the atmosphere.

The term 'visibility' is variously defined in literatures, but generally indicates the distance to which human visual perception is limited by atmospheric conditions. S. Poduri refers to atmospheric visibility as "the clarity with which distant objects are perceived by naked eyes" [1] I. Tombach defines the visibility as "The primarily term based on human perception to be related to the air clarity between the target which is viewed and the observer and also based on the background and target colors, the position of the sun, shadow, light etc., and variety of other physical and psychological factors" [2]. In [3], R. R. Mali describes visibility as "air transparency in the horizontal direction and represents the maximum distance that one can see through the atmosphere at any given time" .

Monitoring of visibility is an important task which needs to be performed precisely and regularly, particularly for the areas sensitive to hazards owing to low visibility like air traffic control, road accidents control and many more. Statistics show that one of the frequent factors leading to accidents is poor visibility (ncrb.gov.in). Poor visibility is also indicative of presence of aerosols that are harmful for health. So, there is a need to develop methods for real time visibility monitoring and in turn supervise air quality. Accordingly, quantitative measures of visibility are increasingly becoming important because quantitative measures are representatives of particles present in the environment that causes degradation of the visibility. Visibility can be quantitatively measured by two parameters namely meteorological range and turbidity. Meteorological range is "The distance under daylight conditions at which the apparent contrast between a black target and its background (horizon sky) becomes equal to a threshold constant of an observer" [4]Turbidity is "the ratio of the optical thickness of a hazed path to the optical thickness of the clean path in atmosphere when there are no molecules"[4].Although turbidity is a great simplification of the true nature of the atmosphere, atmospheric scientists have found it a practical measure of great utility as it does not require complex instrumentation to estimate turbidity.

Currently, the visibility monitoring or air quality estimation processes employed widely can be categorized as optical, view and particle monitoring processes. Optical monitoring methods measure the visibility by looking at optical condition of the atmosphere. Transmissometers and nephelometers are the devices used to monitor the optical quality of the atmosphere [5]-[7].Transmissometers measures how much light can be transmitted from a calibrated incandescent light source through the atmosphere over a known distance. Nephelometers measure the amount of light scattered by gases and particles in a small portion of atmosphere. Particle monitoring detects the pollutants present in the atmosphere using specific sensors. These sensors characterize the chemicals present in the air [8] View Monitoring: Transmissometers and particle monitors only 
sample a small part of the scene while view monitoring uses the photographic system to study the scene as a whole. The photographic system consists of a camera, lens, camera databack and timer. It characterises the appearance of a specific scene and the presence of haze [9]-[17]Hybrid method is the combination of the particle, optical and scene.

The techniques employing fixed camera in scene monitoring methods fail to take observation at night. The instruments deployed for optical monitoring are expensive and need skilled personnel to be operated and capture observations for a limited area. Sensor based techniques employing particle monitoring suffer from high cost of installation and maintenance. The observations are affected by change in field view due to traffic and the sensors need constant power supply. Moreover, sensor installation is sparse and their relocation is also very difficult because of their size and weight. Precision of sensors employed in particle-based monitoring is inconsistent depending on the electric equipment used.

Nowadays mobile phones equipped with high resolution digital cameras, extensive storage capacity, exceptional computing power offers a massive yet ubiquitous infrastructure for various scientific and commercial applications. Additionally, capabilities of smart phone in the form of sensors provides a support to estimate real time parameters like latitude, longitude, orientation, time etc. Motivated from this scenario, this paper proposes a low-cost solution for reporting and mapping turbidity as a quantitative measure of atmospheric visibility. Using the mobile application, real time visibility data can be generated at varied spatial and temporal resolution creating consolidated database for further analysis and decision making by administration. This paper presents the theoretical framework, implementation details, and results of testing and validation.

\section{II.THEORETICAL FRAMEWORK}

Atmospheric turbidity in principle is estimated by matching the scaled luminance obtained from empirical models of sky appearance with observed intensity value of sky image captured through mobile phone camera. The seven well known sky luminance distribution models have been proposed namelyBrunger model, Matsuzawa model, ASRC-CIE model, Perez model, Perraudeau model, Harrison model and Kittler model[5]. Of these models, Perez model is found to work under different weather conditions [18]. Therefore, we zeroed down on implementation of Perez model. During acquisition phase, image of the sky is acquired and the real time parameters like latitude, longitude, time and focal length of camera registered. Android's orientation API's are used to estimate zenith and azimuth angle of the camera. The latitude, longitude and timer are used to estimate the zenith angle and azimuth angle of sun and sky and angle between sun and sky. Using these parameters, sky luminance is determined. This measured luminance is matched with the observed intensity value of the sky element captured. Figure 1 depicts the components utilised to estimate the turbidity.

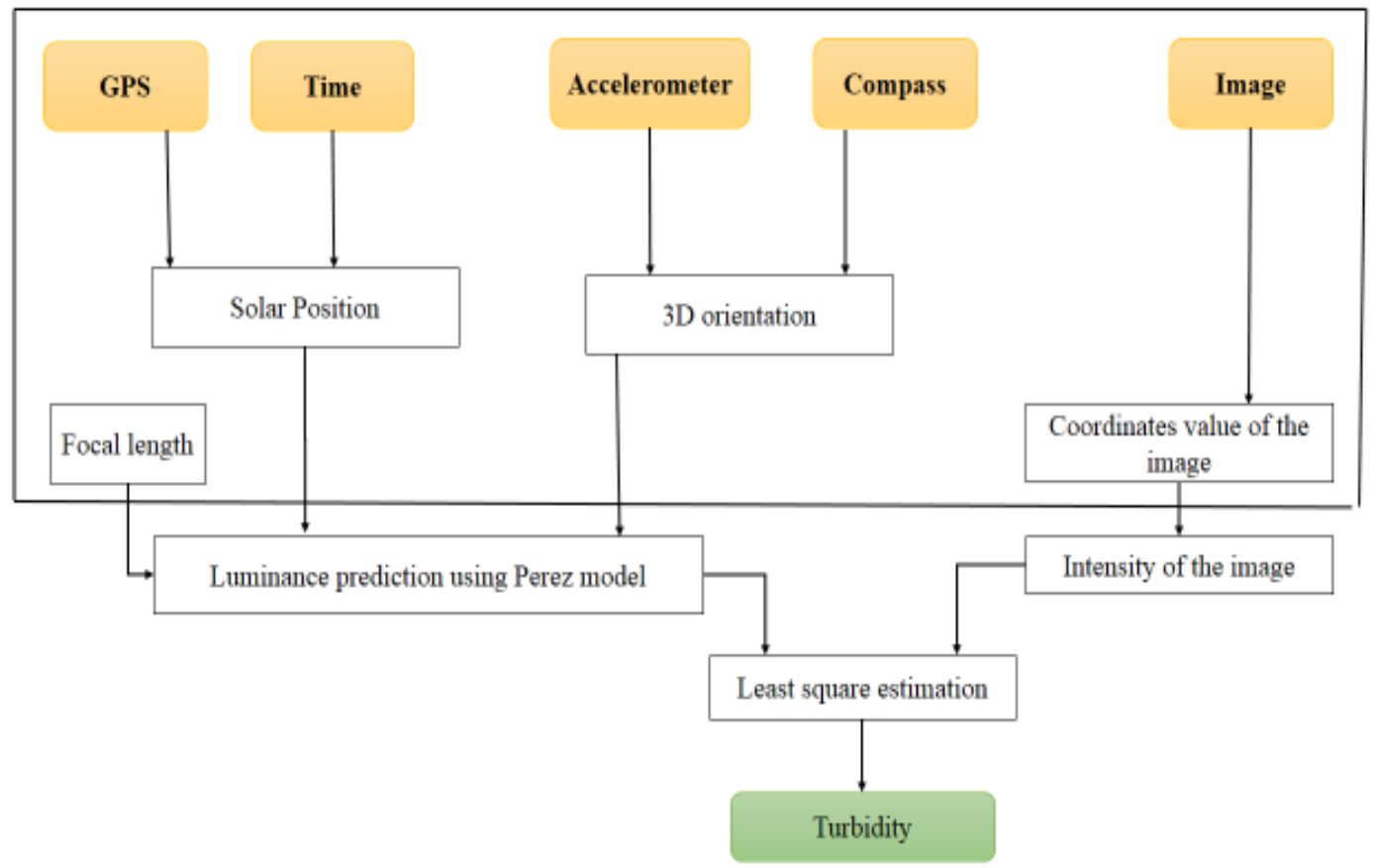

Figure 1: Theoretical framework of proposed application 


\subsection{Implementation of Perez model}

The first step towards luminance determination is finding solar position. The flowchart for the same is shown in Figure 2. The timer of the phone returns local time of the place under observation. The decimal hour of this time is extracted. The location of the place and local time determines the time zone (Equation 1) and Local Standard time meridian (Equation 2). Day of the year is extracted from the date returned by the phone. It is a number between 1 and 365 (for leap year between 1 and 366), 1st January is the day 1. To correct for the eccentricity of the Earth's orbit and the Earth's axial tilt, the equation of time (EoT) (in minutes) is found (Equation 3). To account for the variation of the Local Solar Time (LST) within a given time zone due to the longitude variations within the time zone, time correction is calculated utilising the equation of time (Equation 4).The Local Solar Time (LST) can be found by using the two corrections to adjust the local time (LT) (Equation 5).The local solar time (LST) is converted into the number of degrees which the sun moves across the sky using Hour angle. The hour angle $(\mathrm{H})$ is the angle through which the sun moves across the sky. It takes one hour for the sun to cover $15^{\circ}$ angular distance in the sky. So, the local solar time (LST) can be converted into the hour angle as shown in (Equation 6).The declination of the sun is the angle between the equator and a line drawn from the centre of the Earth to the centre of the sun. It varies seasonally due to the tilt of the Earth on its axis of rotation and the rotation of the Earth around the sun. Accordingly, declination angle is found utilising day of the year (Equation 7). Finally, the declination angle, hour angle and latitude of the place leads to the elevation angle (Equation 8). The elevation angle is the angular height of the sun in the sky measured from the horizontal plane at a location on earth. Zenith angle is the angle between the vertical line at a place on earth and the line joining the place to the sun. It is calculated as complement of elevation angle (Equation 9). The azimuth angle is the compass direction from which the sunlight is coming. Elevation angle, declination angle and latitude of place determines the azimuth (Equation 10). The zenith angle $\theta c$ and azimuth angle $\varphi c$ of the camera are calculated by sensors in the camera. The azimuth and zenith of sky element and relative orientation to solar position $\gamma \mathrm{p}$ are given by Equation 11, 12 and 13 respectively. The relative luminance lp of a sky element is a function of its zenith angle $\theta p$ and the angle $\gamma p$ with the sun and is given by Equation 14 Where the 5 constants a, b, c, d, e specify the current atmospheric conditions, and all angles are expressed in radians [19]. Each of the parameters has a specific physical effect on the sky distribution and with different values they can capture a wide variety of sky conditions. For clear skies, the constants take on the following values: $a=-1$, $\mathrm{b}=-0.32, \mathrm{c}=10, \mathrm{~d}=-3, \mathrm{e}=0.45$. Scaled luminance (Equation 16) is evaluated as ratio of true luminance to zenith luminance (Equation 15).

\subsection{Turbidity estimation}

Visibility is estimated by matching the scaled luminance ratio (f) with the observed image intensity values at sky pixels Intensity $\mathrm{I}$ is computed from RGB values using the CIE standard formula $\mathrm{I}=0.2126 \mathrm{R}+0.7152 \mathrm{G}+0.0722 \mathrm{~B}$. Turbidity $\mathrm{t}$ is estimated as the value that minimizes the sum of squared error between measured intensity and the analytic luminance value as obtained from Perez model over the set $\mathrm{P}$ of sky pixels
(Equation 18). $\mathrm{k}$ is the constant factor between the image intensity $I$ and $f$ at each pixel.

\section{III.IMPLEMENTATION ENVIRONMENT}

The application calculates the visibility in the form of turbidity. The turbidity encodes the amount of scattering in the atmosphere, so the lower the $t$, the clearer the sky. On execution of application the welcome screen with the tabs for capturing the scene, help and viewing history data FIGURE 4An alert to put on the GPS of the phone appears if it is off Figure 5(a) Location can be implicitly taken by GPS of the phone or can be manually entered by the user by interactively selecting from the phone Figure 5(b). To ensure that only the sky image is taken, the image is captured at an orientation angle greater than $130^{\circ}$. The capture button will be activated only at this angle The user is provided with the option to capture the relevant area of the scene captured i.e., facility to crop the image is provided FIGURE 6(a). The application calculates turbidityFIgURE 6(b), plots histogram of the scene and provides the option to share the information. The results can be stored on the application locally and can be uploaded to the web server Figure 8 .

Features of application

- Implicitly act as solar calculator

- Data Sharing: The results can be instantaneously shared amongst internal application as well as contacts

- Communication: The data transfer is initiated immediately after assuming that the phone is connected to the internet.

- Aiding data collection: Sensors on the phone guide data collection so that the data is well-suited for the estimation algorithm. Like the camera button is activated only if the zenith angle is more than $100^{\circ}$ as we are only interested in images of the sky.

- Human centric: When the use captures an image, we ask him to select a part of the image that is sky.

- Energy efficiency: Only GPS and camera are the sensors consuming energy

- Citizen Science: The application can be deployed over various devices and common public can be involved to take observations at spatial and temporal resolutions 
Table 1: summary of Equations used

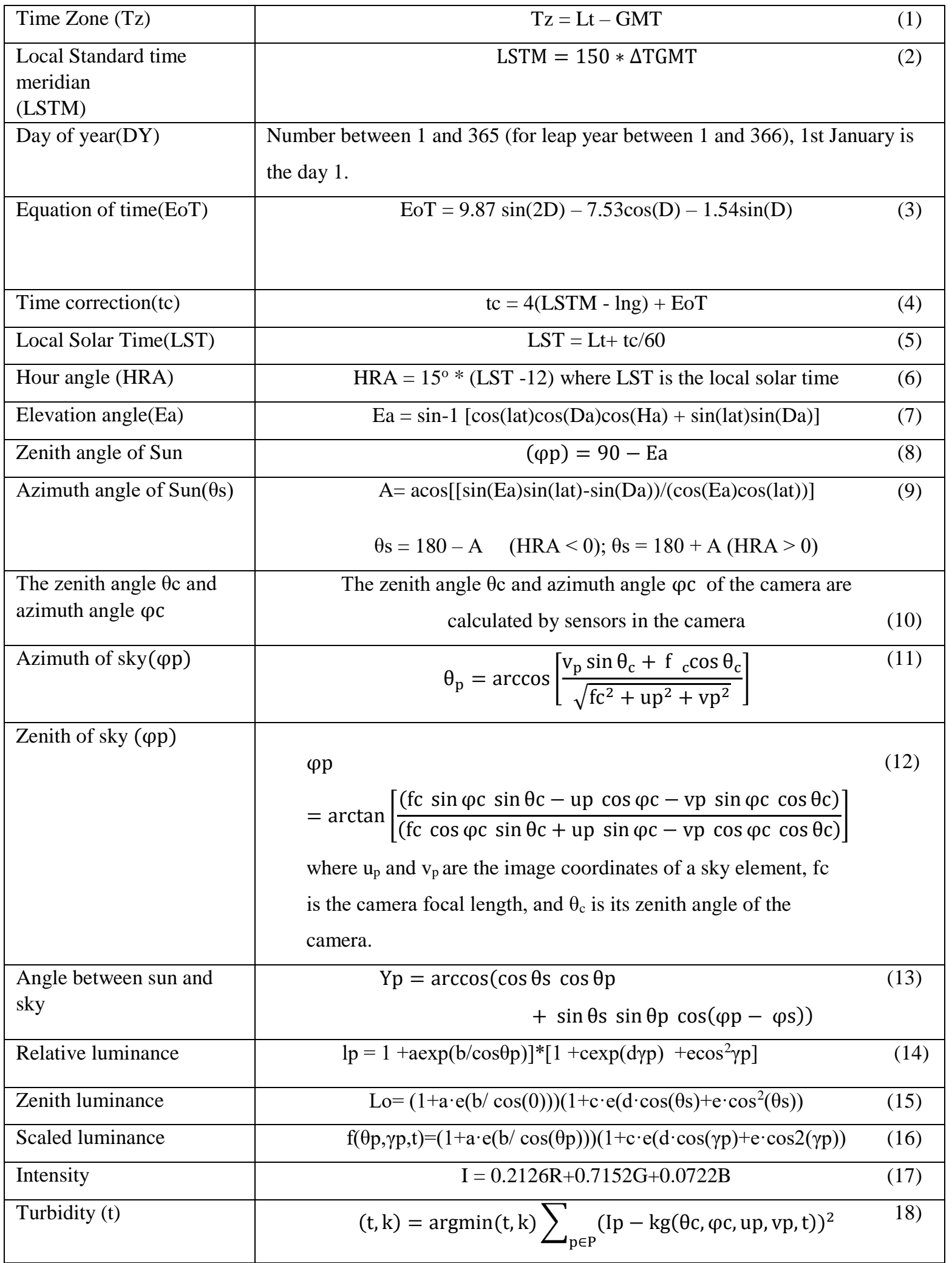




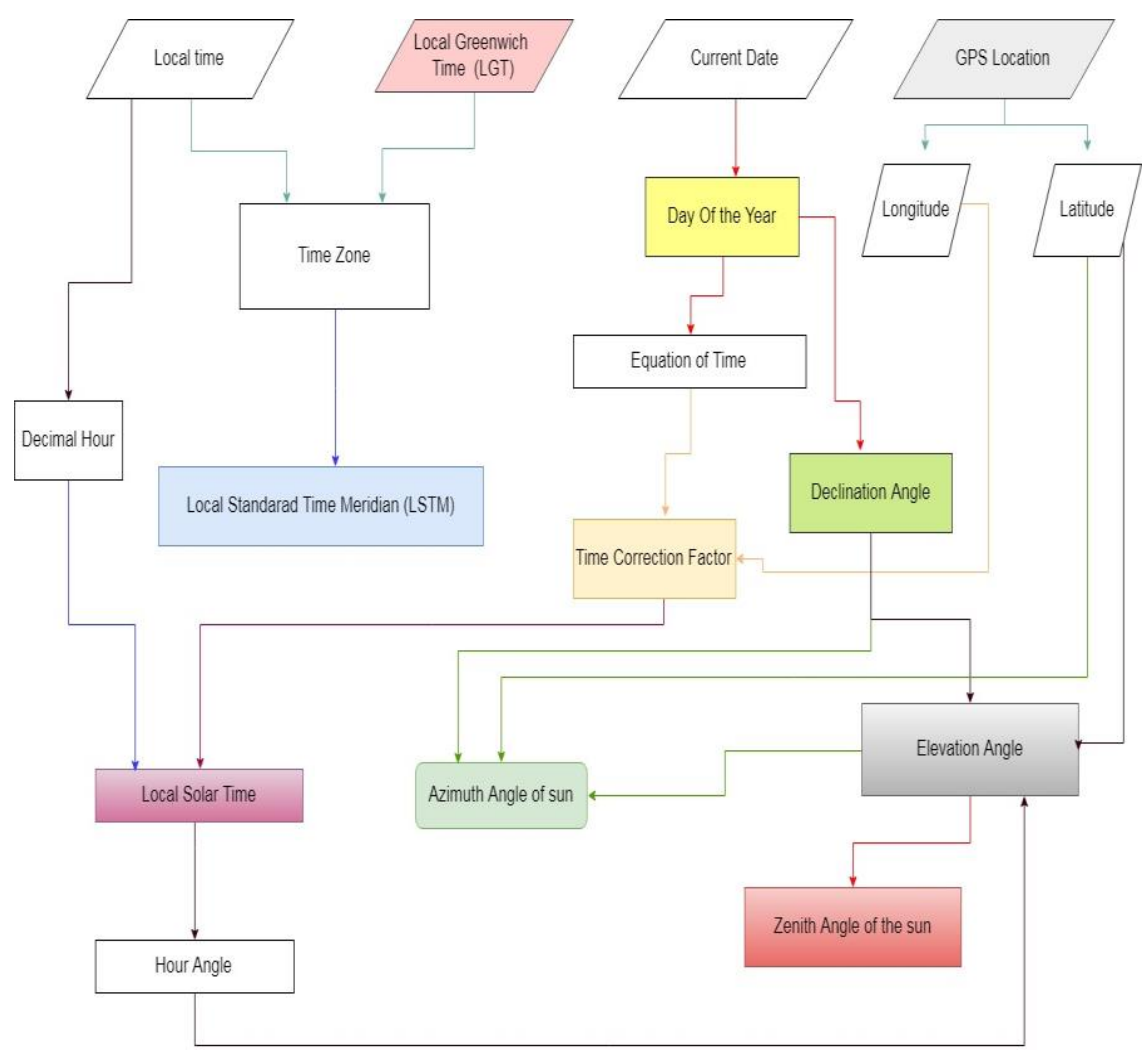

FIGURE 2: FLOWCHART FOR CALCULATION OF SUN'S AZIMUTH AND ZENITH

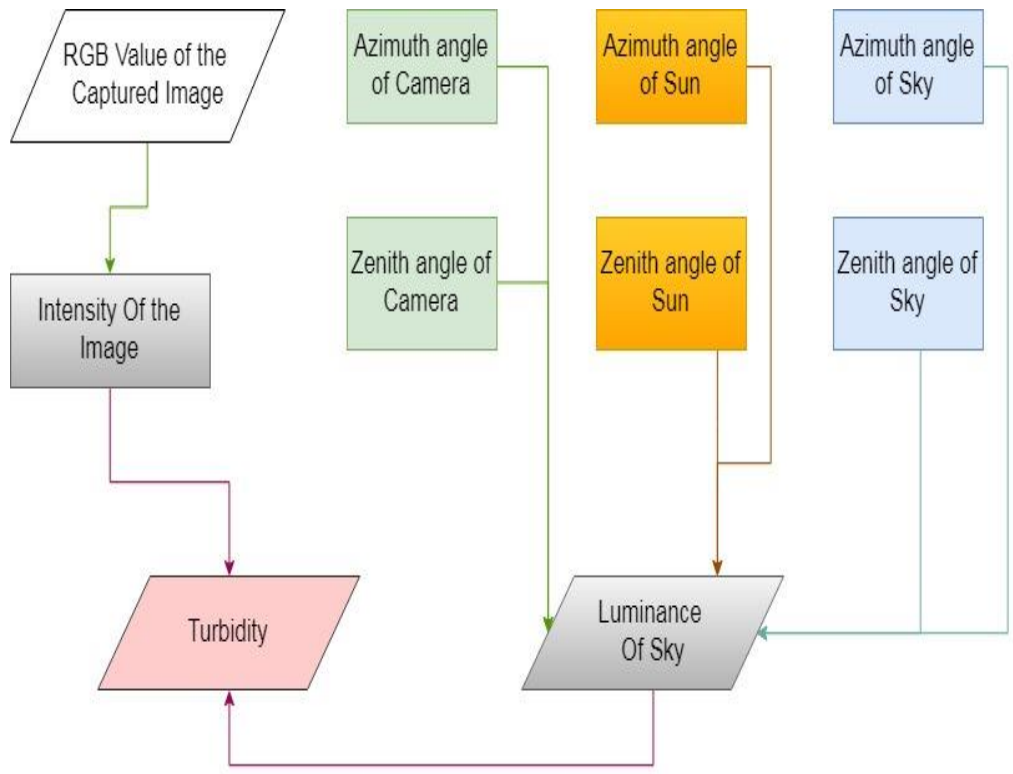

FIGURE 3: TURBIDITY ESTIMTION 


\section{IV.IMPLEMENTATION ENVIRONMENT}

The application calculates the visibility in the form of turbidity. That is the lesser the visibility the more is the visibility and vice versa. On execution of application the welcome screen with the tabs for capturing the scene, help and viewing history data appears FIGURE 4. An alert to put on the GPS of the phone appears if it is off Figure 5(a). Location can be implicitly taken by GPS of the phone or can be manually entered by the user by interactively selecting from the phone Figure 5(b). To ensure that only the sky area is captured, the capture button is activated only when the orientation angle of the camera is around 130 degree. Cropping facility is provided as an option to select a relevant area of the captured image FIGURE 6(a). Results are calculated in the form of turbidity and the implication of that turbidity level to health Figure 6(b). The application calculates turbidity, plots histogram of the scene and provides the option to share the information. The results can be stored on the application locally and can be uploaded to the web server for mapping on google maps Figure 8 as well as generate analytical reports. Information regarding use of application is provided under help tab and history tab provides archive of previous observations Figure 7.

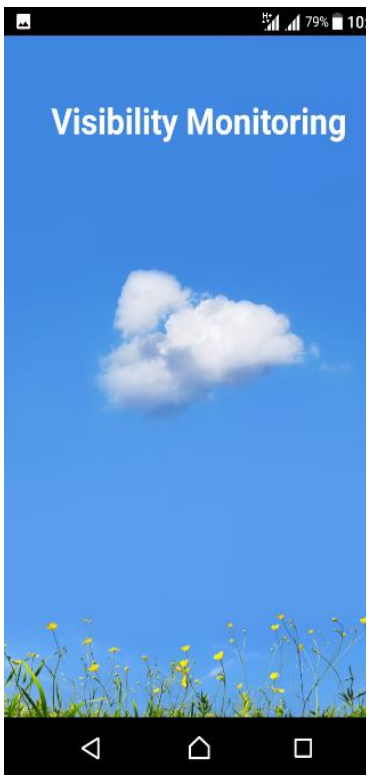

(a)

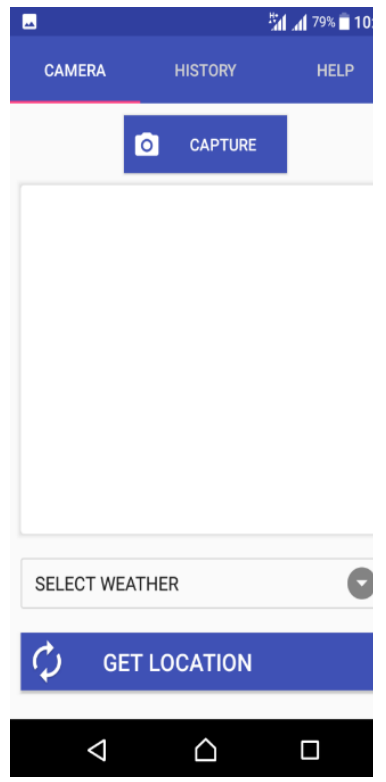

(b)
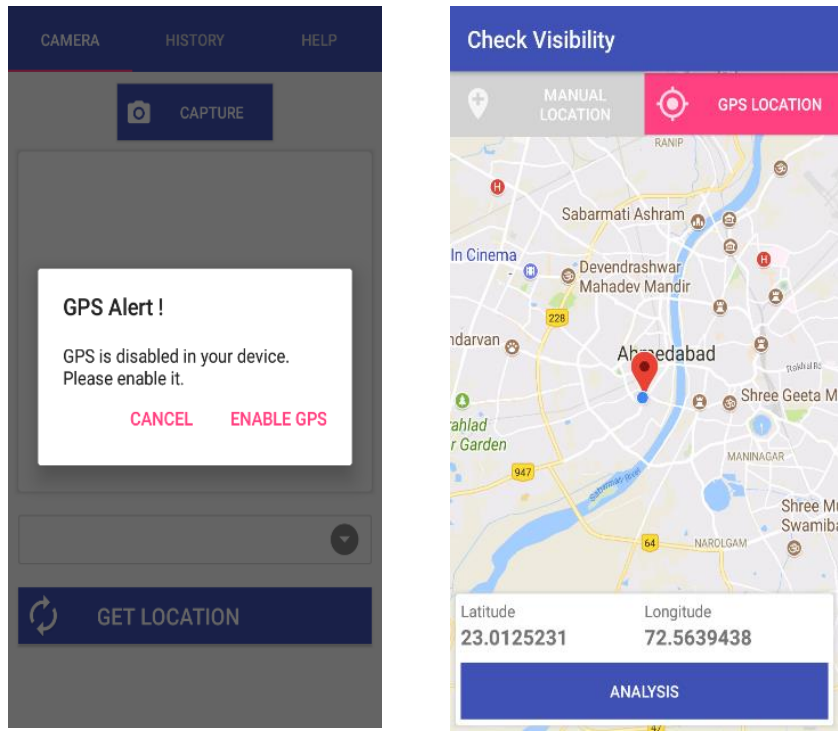

(a)

(b)

Figure 5: (a) GPS alert (b) Interactive selection of the location
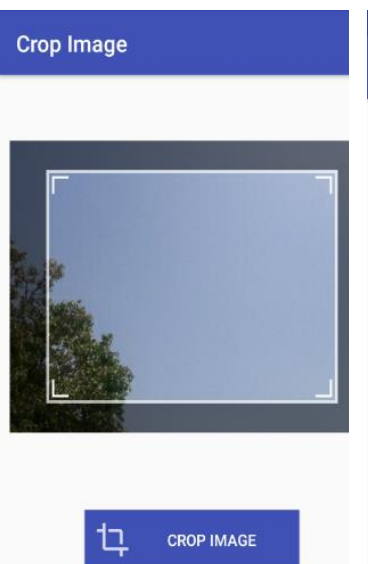

GO WITHOUT CROP

(a)

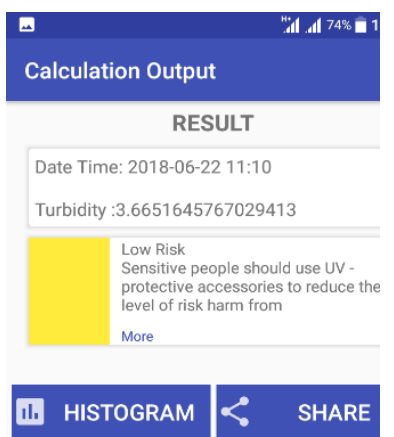

II. HISTOGRAM
FIGURE 4: HOME SCREEN 


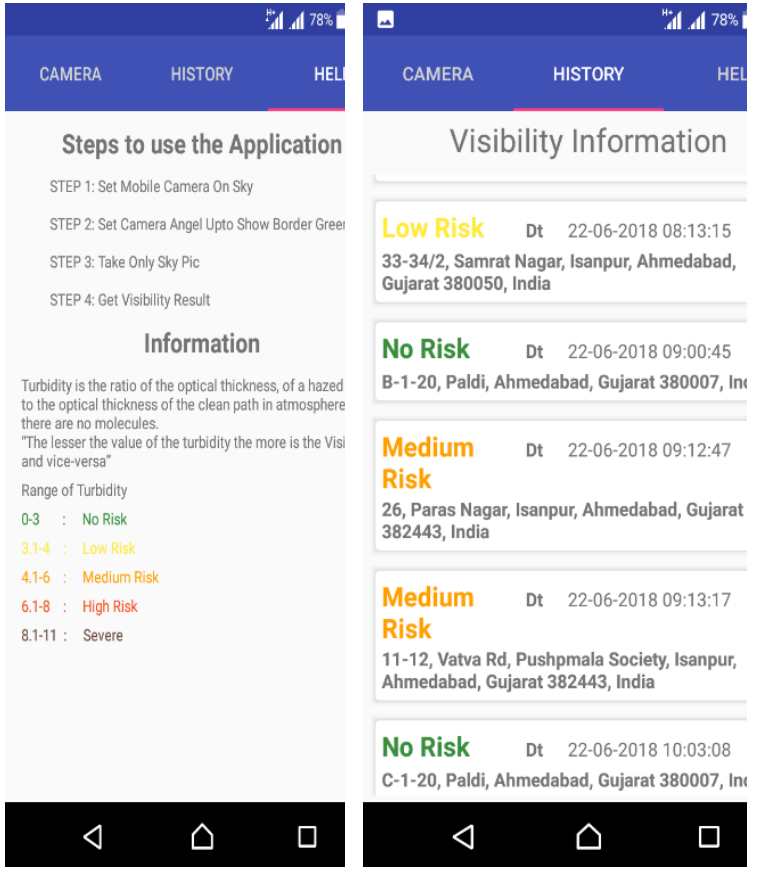

(a)

(b)

FIGURE 7: (a) Help Screen (b) History of captured results

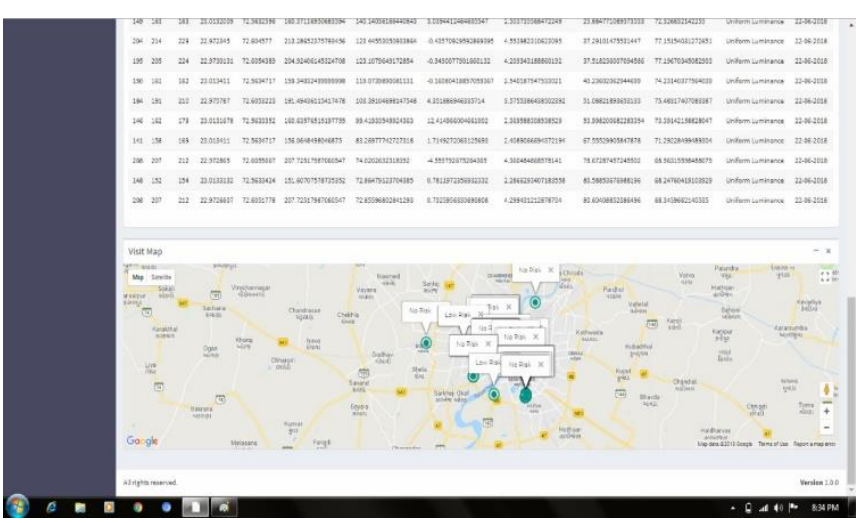

Figure 8: Turbidity mapping on Google maps

\section{V.RESULTS}

Since no ground truth turbidity data was available, the correction of the results of the application were validated by following methods:-

(1) Validation of intermediate results obtained during the implementation of Perez model namely time zone, local standard time meridian, declination angle, elevation angle, zenith angle, azimuth angle were verified by websources like NOAA Solar Calculator.

(2) Result of turbidity calculation were validated by observing correlation with Air Quality Index observed from different SAFAR stations at Ahmedabad.

(3) The dependence of camera and sensor on observations was observed by taking concurrent applications from different phones.

(4) Observations were taken during different weather conditions and areas with different contamination levels to observe the variability in turbidity.

Test drives of application was conducted during the months of April,May and June for the city of Ahmedabad. April and May being the months with clear sky conditions and June with commencement of Monsoon, with cloudy sky conditions. The conclusions from observations are as follows:

i. Azimuth and Zenith angles calculated from our application were found to be consistent with the the web sources with accuracy ranging from $96 \%$ to $100 \%$ at different times of day. Comparitive plot of observations for the day 23/04/2018 at irregular time intervals is shown in Figure 9 and Figure 10. Shows the pattern of zenith angle for the date 23/04/2018 at regular intervals of 30 minutes. The minimum value is 13 deegrees at 12 p.m. where the elevation of sun is maximum at 78 degrees.

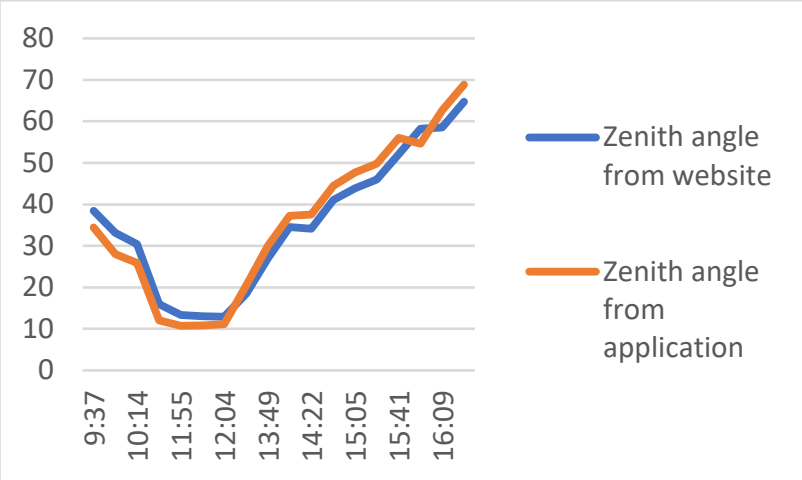

Figure 9: Comparative plot of Zenith angle

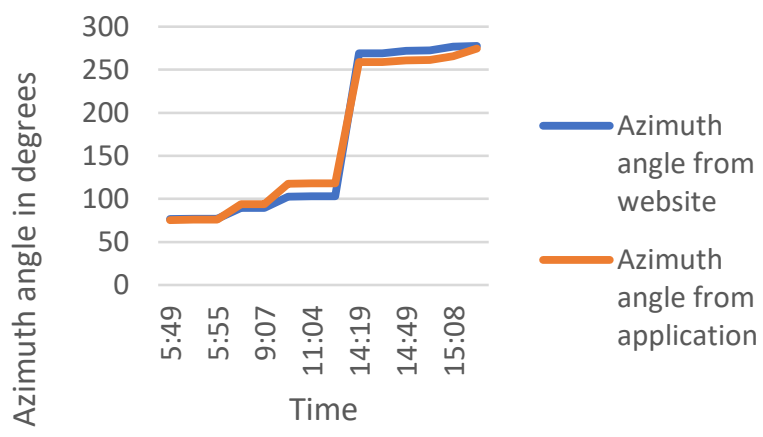

Figure 10: Comparative plot of Azimuth angle

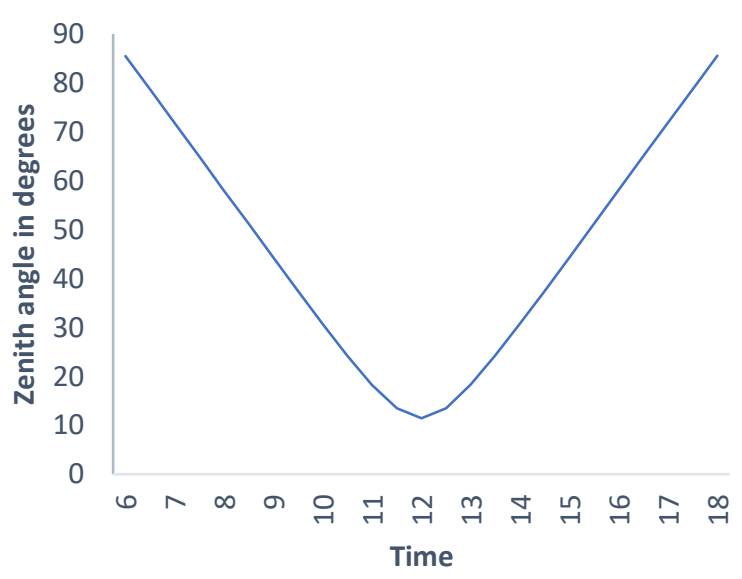

Figure 11: Zenith angle observed from 6 a.m to 6 p.m.

ii. A test drive for observing the varition of observation with mobile phone was performed 
on 01/05/2018 using three different phones with varying camera specifications. The phones used were Moto Xplay(20 MP), Lg-L90(8 MP) and Samsung J7(24 MP). Though the pattern of turbidity obtained was same for the three phones, individual turbidity captured was found to be correlated with megapixels of the camera Figure 12.

iii. Test drive to observe correlation of turbidity with place was performed on 26/04/2018 with Samsung J7 phone. Areas with variable pollution levels were covvered. The range of turbidity observed was only 1.2. Highest turbidity was observed at $100 \mathrm{ft}$ ring road cluttered with traffic and lowest at a small street area of Prernatirth derasar Figure 13.

iv. Another test drive was performed on 21/06/2018 to find correlation between turbidity values of application and Air Quality Index reported at seven SAFAR stations of Ahmedabad by taking concurrent observations from stations as well as application. A correlation of $96 \%$ was observed Figure 14.

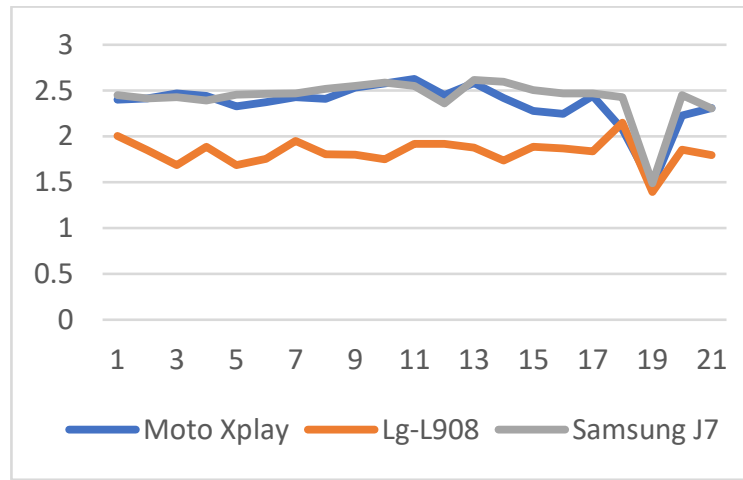

Figure 12: observations with different mobile phones

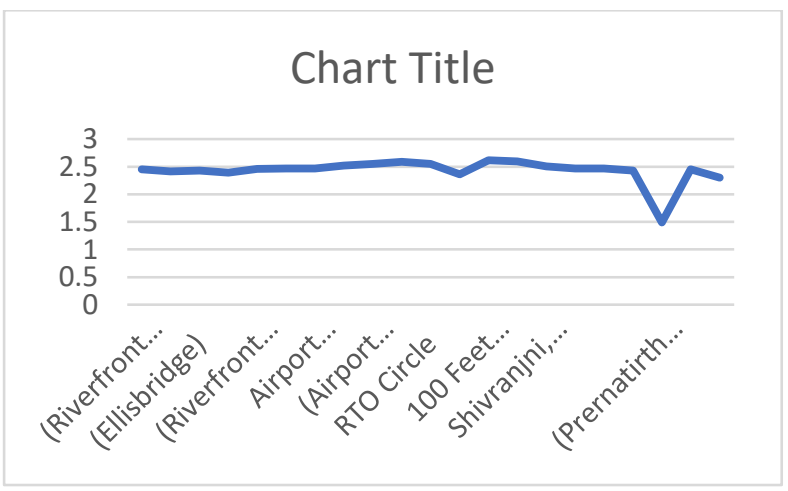

Figure 13: Observations at different locations

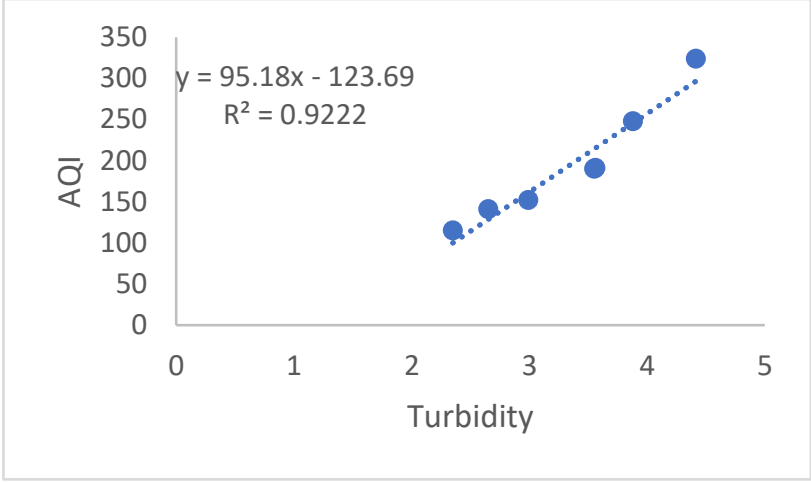

Figure 14: Turbidity vs Air Quality Index

v. Variability of turbidity with weather condition was observed by taking the observations under different climatic conditions. During the months of April and May with clear sky conditions, Turbidity was observed in the range of 1 to 2.5 . However in the month of June, at onset of Monsoon with cloudy weather, Turbidity value of 5.27 was obtained. Shows the two sky conditions.

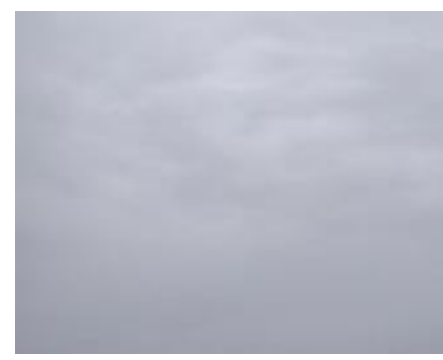

Figure 15: Cloudy sky with Turbidity 5.27

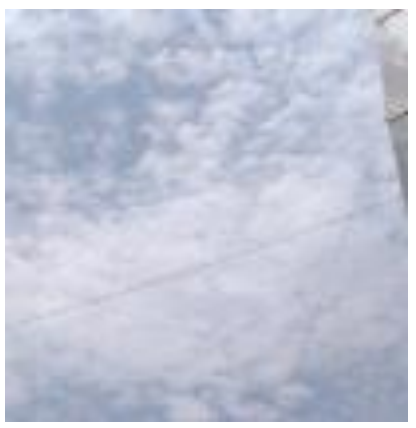

Figure 16: Clear sky with Turbidity 1.2 


\section{VI.CONCLUSION}

A mobile application to measure atmospheric turbidity has been developed and teseted under different weather conditions, locations, devices. The results validated by comparing with air Quality index observed at different SAFAR stations of Ahmedabad city. The application was found to be consistent and accurate.

\section{ACKNOWLEDGEMENT}

We would like to thank Ms. Neha Rajput and Ms. Shrushti for helping us in coding and testing of the application

\section{REFRENECES}

[1] S. Poduri, A. Nimkar, and G. S. Sukhatme, "Visibility monitoring using mobile phones," Annu. Rep. Cent. Embed. Networked Sens., pp. $125-127,2010$.

[2] I. Tombach and D. Allard, "Intercomparison of visibility measurement methods," J. Air Pollut. Control Assoc., vol. 30, no. 2, pp. 134-142, 1980.

[3] R. R. Mali and R. D. Vashistha, "Visibility Measurement technique and Its Application in Aviation Services at International Airports in India." .

[4] E. J. McCartney, "Optics of the atmosphere: scattering by molecules and particles," New York, John Wiley Sons, Inc., 1976. 421 p., 1976.

[5] P. Ineichen and B. Molineaux, "Characterisation and comparison of two sky scanners: PRC Krochmann and EKO instruments," in First draft, IEA Task XVII expert meeting, 1993.

[6] L. W. Richards, "Sight path measurements for visibility monitoring and research," JAPCA, vol. 38, no. 6, pp. 784-791, 1988.

[7] S. NathanGraves, "Usingvisibilitycamerasto estimateatmosphericlightextinction," Appl. Computerv., p. r584, 2011.

[8] J. F. Sisler and W. C. Malm, "Interpretation of Trends of PM25 and Reconstructed Visibility from the IMPROVE Network," J. Air Waste Manage. Assoc., vol. 50, no. 5, pp. 775-789, 2000.

[9] L. Xie, A. Chiu, and S. Newsam, "Estimating atmospheric visibility using general-purpose cameras," in International Symposium on Visual Computing, 2008, pp. 356-367.

[10] D. Bäumer, S. Versick, and B. Vogel, "Determination of the visibility using a digital panorama camera," Atmos. Environ., vol. 42, no. 11, pp. 2593-2602, 2008.

[11] R. Babari, N. Hautière, É. Dumont, N. Paparoditis, and J. Misener, "Visibility monitoring using conventional roadside cameras-Emerging applications," Transp. Res. part C Emerg. Technol., vol. 22, pp. 17-28, 2012.

[12] N. Hautiére, R. Babari, É. Dumont, R. Brémond, and N. Paparoditis, "Estimating meteorological visibility using cameras: a probabilistic modeldriven approach," in Asian Conference on
Computer Vision, 2010, pp. 243-254.

[13] F. M. Caimi, D. M. Kocak, and J. Justak, "Remote visibility measurement technique using object plane data from digital image sensors," in Geoscience and Remote Sensing Symposium, 2004. IGARSS'04. Proceedings. 2004 IEEE International, 2004, vol. 5, pp. 3288-3291.

[14] Y. Li, J. Huang, and J. Luo, "Using user generated online photos to estimate and monitor air pollution in major cities," in Proceedings of the 7th International Conference on Internet Multimedia Computing and Service, 2015, p. 79.

[15] L. W. Richards, M. Stoelting, and R. G. M. Hammarstrand, "Photographic method for visibility monitoring," Environ. Sci. Technol., vol. 23, no. 2, pp. 182-186, 1989.

[16] F. M. Janeiro, F. Wagner, P. M. Ramos, and A. M. Silva, "Automated atmospheric visibility measurements using a digital camera and image registration," in 1st IMEKO TC19 International Symposium on Measurements and Instrumentation for Environmental Monitoring, 2007, pp. 254-259.

[17] R. K. O. Bayot, R. T. Labuguen, E. J. P. Volante, N. J. C. Libatique, and G. L. Tangonan, "Urban visibility measurements during tropical weather events using image processing," in TENCON 2012-2012 IEEE Region 10 Conference, 2012, pp. 1-6.

[18] R. Perez, R. Seals, and J. Michalsky, "Allweather model for sky luminance distributionpreliminary configuration and validation," Sol. energy, vol. 50, no. 3, pp. 235-245, 1993.

[19] P. Ineichen, B. Molineaux, and R. Perez, "Sky luminance data validation: comparison of seven models with four data banks," Sol. Energy, vol. 52, no. 4, pp. 337-346, 1994. 